Brief paper

\title{
A model predictive control approach to the periodic implementation of the solutions of the optimal dynamic resource allocation problem
}

\author{
Jiangfeng Zhang, Xiaohua Xia* \\ Centre of New Energy Systems, Department of Electrical, Electronic and Computer Engineering, University of Pretoria, Pretoria 0002, South Africa
}

\section{A R T I C L E I N F O}

\section{Article history:}

Received 21 November 2009

Received in revised form

5 May 2010

Accepted 21 September 2010

Available online 27 November 2010

\section{Keywords:}

Model predictive control

Perfect optimal dynamic resource

allocation problem

Energy optimization

\begin{abstract}
A B S T R A C T
This paper proposes a model predictive control (MPC) approach to the periodic implementation of the optimal solutions of a class of resource allocation problems in which the allocation requirements and conditions repeat periodically over time. This special class of resource allocation problems includes many practical energy optimization problems such as load scheduling and generation dispatch. The convergence and robustness of the MPC algorithm is proved by invoking results from convex optimization. To illustrate the practical applications of the MPC algorithm, the energy optimization of a water pumping system is studied.
\end{abstract}

(C) 2010 Elsevier Ltd. All rights reserved.

\section{Introduction}

There is a particular class of problems in resource allocation and energy appliance scheduling pertaining to special periodic constraints in minimizing objective functions. This class of problems includes the scheduling of appliances (pumps, conveyor belts, generators, etc.) on a daily or weekly basis. The demand, operation conditions, and constraints change only periodically during this time. For the purpose of this paper, this class of problems are called Optimal Dynamic Resource Allocation Problems (ODRAP). Such problems will usually be solved over one period, e.g. $24 \mathrm{~h}$, and the optimal solution will be repeatedly implemented for other periods without considering interactions between periods (Middelberg, Zhang, \& Xia, 2009). The interaction between periods of implementation is what makes the ODRAP different from those resource allocation and scheduling problems generally studied (see Aissi, Bazgan, and Vanderpooten (2009), Belfares, Klibi, Lo, and Guitouni (2007), Biegler and Zavala (2009), Ibaraki and Katoh (1988), Lee, Kumara, and Gautam (2007), Lee and Lee (2006), Munawar and Gudi (2005), Pasadyn, Lee, and Edgar (2008), Patriksson (2008) and Zafra-Cabeza, Ridao, and Camacho (2008) and the references therein). In these papers, the research is focused on developing various algorithmic approaches to resource allocation problems

\footnotetext{
The material in this paper was not presented at any conference. This paper was recommended for publication in revised form by Associate Editor Lalo Magni under the direction of Editor Frank Allgöwer.

* Corresponding author. Tel.: +27 12 4202165; fax: +27 123625000 .

E-mail addresses: zhang@up.ac.za (J. Zhang), xxia@postino.up.ac.za (X.Xia).
}

in which periodic constraints may not exist. Even though periodic constraints are included in the problem, the corresponding backgrounds are either not applicable to ODRAP or not explicitly discussed. A lack of discussion particularly pertaining to the periodic implementations of optimal solutions for periodic resource allocation problems exists. There may, in fact, be unintended interactions between two periods when an optimal solution of an ODRAP for one period is implemented over consequent periods. A ramp rate violation, for example, may occur when the optimal solution of the dynamic economic dispatch (DED) problem over the first day (Xia, Zhang, \& Elaiw, 2009) is simply implemented in the second day. This ramp rate violation actually implies that the constraints in an ODRAP formulation for DED are inadequate for periodic implementation. This problem is solved by introducing more constraints and thus formulating a new ODRAP, or a Perfect Optimal Dynamic Resource Allocation Problem (PODRAP). Furthermore, Xia et al. (2009) also proposes an algorithm to solve this revised dynamic economic dispatch problem by the model predictive control (MPC) approach. The same approach will be extended to the general PODRAP in this paper.

The ability of the MPC to handle constraints, being able to use simple models, and its closed-loop stability and inherent robustness makes it very practical for use in industrial problems (see Allgöwer and Zheng (2000), Garcia, Prett, and Morari (1989), and Qin and Badgwell (2003)). This MPC approach is also applied in general resource allocation or scheduling problems as done in Ferrari-Trecate et al. (2004), Lee et al. (2007), Lee and Lee (2006), Munawar and Gudi (2005), van Staden, Zhang, and Xia (2009), and Zafra-Cabeza et al. (2008). It is interesting to note that MPC algorithms have a close relationship with optimization since an 
MPC algorithm needs to solve an optimization problem in each iteration. Studies on the connections of MPC with optimization were conducted since the 1960's (see Chang and Seborg (1983) and Zadeh and Whalen (1962)). Modern MPC approaches for resource allocation problems do not take the relationship of the MPC solutions and the global optimal resource allocation solutions into consideration.

The aim of this paper is to develop an MPC algorithm for the periodic implementation of the optimal solutions of the PODRAP. Further it will prove the algorithm's convergence and the corresponding robustness against disturbances in controller implementation or state measurement. The convergence result reveals that the optimal solutions in the MPC algorithm converge to the optimal solution of the PODRAP. The formulation of PODRAP and the perfection of ODRAP can be found in Section 2, while the convergence and robust results are in Section 3. An example on the voluntary load shedding problem for a water pumping system is also studied in Section 4 to illustrate the formulation of a PODRAP and the convergence and robustness of the MPC approach. Some concluding remarks are drawn in the last section.

The following nomenclatures are fixed.

$\sum_{i=j}^{k} a_{i}$ equals 0 for $j>k$ and $a_{j}+\cdots+a_{k}$ if $j \leq k$;

$\operatorname{col}\left(\alpha_{1}, \alpha_{2}, \ldots, \alpha_{s}\right)$ equals $\left(\alpha_{1}^{T}, \alpha_{2}^{T}, \ldots, \alpha_{s}^{T}\right)^{T}$ for any column

vectors $\alpha_{1}, \alpha_{2}, \ldots, \alpha_{s}$;

s.t.: subject to;

$m, p, n$ : fixed positive integers

$k, j$ : the $k$-th or $j$-th sampling time interval;

$(k, k+p$ ]: the time interval from $k$ to $k+p$ excluding $k$;

$X_{i}^{j}$ : a 1-dimensional real variable for all $i, j$;

$X[j]$ : the $m$-dimensional real vector variable for all $j$;

$X[k \mid j]$ : the prediction of $X[k]$ at time $j$ for any $k \geq j$;

$Z[k]$ : equals $\operatorname{col}(X[k], X[k+1], \ldots, X[k+p-1])$;

$Z[k \mid j]$ : the prediction of $Z[k]$ at time $j$ for any $k \geq j$;

$u_{k}^{j}$ : equals $X[k+1 \mid j]-X[k \mid j]$;

$u[k \mid j]:=\operatorname{col}\left(u_{k}^{j}, u_{k+1}^{j}, \ldots, u_{k+p-1}^{j}\right)=z[k+1 \mid j]-z[k \mid j] ;$

$i_{1}, i_{2}, \ldots, i_{s}$ : integers with $1 \stackrel{k+p-1}{\leq} i_{1}<i_{2}<\cdots<i_{s}=n$;

$x:=\left(x_{1}, x_{2}, \ldots, x_{n}\right) \in \mathbb{R}^{n}$;

$y^{\ell}:=\left(x_{i_{\ell-1}+1}, x_{i_{\ell-1}}, \ldots, x_{i_{\ell}}\right), \ell=1,2, \ldots, s$, are called

partitions of $x$ such that $x=\operatorname{col}\left(y^{1}, y^{2}, \ldots, y^{s}\right)$.

\section{Problem formulation}

PODRAP $(k, k+p]$ For any give system dynamics $X[k+1]=$ $G(X[k])$, a PODRAP over the time interval $(k, k+p]$ is defined as the minimization problem:

$\min J_{k+1}(z[k+1 \mid k+1])$

s.t. $H_{k+1}(z[k+1 \mid k+1]) \geq 0$,

where the optimization variable is $z[k+1 \mid k+1]=\operatorname{col}(X[k+$ $1 \mid k+1], X[k+2 \mid k+1], \ldots, X[k+p \mid k+1])$, and $J_{k+1}$ and $H_{k+1}$ are smooth, convex functions satisfying the following periodic invariant properties for all $k \geq 0$ :

- Periodic invariant objective:

$$
\begin{aligned}
& J_{k+1}(X[k+1], X[k+2], \ldots, X[k+p]) \\
& \quad=J_{k+2}(X[k+2], \ldots, X[k+p], X[k+1]),
\end{aligned}
$$

- Periodic invariant constraints:

$$
\begin{aligned}
&\{(X[k+1], X[k+2], \ldots, X[k+p]): \\
& \quad\left.H_{k+1}(X[k+1], X[k+2], \ldots, X[k+p]) \geq 0\right\} \\
&=\{(X[k+1], X[k+2], \ldots, X[k+p]): \\
&\left.H_{k+2}(X[k+2], X[k+3], \ldots, X[k+p], X[k+1]) \geq 0\right\} .
\end{aligned}
$$

Definition 1. Fix the notations in $\operatorname{PODRAP}(k, k+p]$, then the minimization problem (1) is called an optimal dynamic resource allocation problem (ODRAP) if the functions $J_{\ell}$ and $H_{\ell}$ are smooth, convex, and $J_{\ell}$ satisfies the periodic invariant property (2) for all $\ell \geq 1$.

Note that the only way in which the ODRAP and PODRAP differ is that an ODRAP may not satisfy the constraints in (3). A practical resource allocation problem may be an ODRAP but not a PODRAP, and the constraints can often be reasonably extended so that the periodic invariant property is satisfied (Xia et al., 2009).

The following two propositions are easy to verify and the proofs are omitted.

Proposition 1. Assume that there exists smooth and convex functions $\alpha_{i}, \beta_{i}, i \geq 1$, such that $\alpha_{i+p} \equiv \alpha_{i}, \beta_{i+p} \equiv \beta_{i}$, and $J_{k+1}$ and $H_{k+1}$ have the following special form:

$$
\begin{aligned}
& J_{k+1}(X[k+1 \mid k+1], \ldots, X[k+p \mid k+1]) \\
& =\sum_{i=k+1}^{k+p} \alpha_{i}(X[i \mid k+1]), \\
& H_{k+1}(X[k+1 \mid k+1], \ldots, X[k+p \mid k+1]) \\
& =\sum_{i=k+1}^{k+p} \beta_{i}(X[i \mid k+1]) .
\end{aligned}
$$

Then the following optimization problem is a PODRAP over the time period $(k, k+p]$

$$
\begin{array}{ll}
\min & J_{k+1}(z[k+1 \mid k+1]) \\
\text { s.t. } & H_{k+1}(z[k+1 \mid k+1]) \geq 0 .
\end{array}
$$

Proposition 2. Assume that $J$ and $H$ are symmetric functions in the sense that $J(X[k+1 \mid k+1], \ldots, X[k+p \mid k+1])=J(X[\sigma(k+$ $1) \mid k+1], \ldots, X[\sigma(k+p) \mid k+1])$ and $H(X[k+1 \mid k+1], \ldots, X[k+$ $p \mid k+1])=H(X[\sigma(k+1) \mid k+1], \ldots, X[\sigma(k+p) \mid k+1])$ hold for any permutation $\sigma$ of $(k+1, k+2, \ldots, k+p)$, then the following optimization problem is a PODRAP over the time period $(k, k+p]$ :

$$
\begin{array}{ll}
\min & J(z[k+1 \mid k+1]) \\
\text { s.t. } & H(z[k+1 \mid k+1]) \geq 0 .
\end{array}
$$

Definition 2. Consider the following optimization problem over the time period $(k, k+p]$

$\min J_{k+1}(z[k+1 \mid k+1])$

s.t. $\quad H_{k+1}(z[k+1 \mid k+1]) \geq 0$,

where $J_{k+1}$ and $H_{k+1}$ are convex and smooth, and $J_{k+1}$ satisfies (2) so that (4) is an ODRAP. Denote as $\Omega_{k+1}$ the feasible domain $\left\{z[k+1 \mid k+1]: H_{k+1}(z[k+1 \mid k+1]) \geq 0\right\}$, and as $\Omega_{k+1}^{\prime}$ the maximum subset of $\Omega_{k+1}$ such that

$$
\begin{aligned}
\Omega_{k+1}^{\prime}:= & \left\{z[k+1 \mid k+1]: H_{k+1}(z[k+1 \mid k+1]) \geq 0,\right. \\
& \left.H_{k+1}^{\prime}(z[k+1 \mid k+1]) \geq 0\right\},
\end{aligned}
$$

with $H_{k+1}^{\prime}$ a convex and smooth function, and the function $\operatorname{col}\left(H_{k+1}, H_{k+1}^{\prime}\right)$ satisfying the periodic invariant property (3). Then the following minimization problem over $(k, k+p]$ is called a perfection of the ODRAP (4):

$$
\begin{array}{ll}
\min & J_{k+1}(z[k+1 \mid k+1]) \\
\text { s.t. } & H_{k+1}(z[k+1 \mid k+1]) \geq 0, \quad H_{k+1}^{\prime}(z[k+1 \mid k+1]) \geq 0 .
\end{array}
$$

Obviously the perfection of an ODRAP is a PODRAP. The following result constructs a perfection for an ODRAP. The proof is straightforward and thus omitted. 
Proposition 3. Fix the notations in Definition 2 and consider the ODRAP in (4). Let

$$
\begin{aligned}
\Omega_{k+1}^{\prime}= & \left\{z[k+1 \mid k+1]: H_{k+i p+1}(X[k+1], X[k+2], \ldots,\right. \\
& X[k+p]) \geq 0, H_{k+i p+2}(X[k+2], X[k+3], \ldots, \\
& X[k+p], X[k+1]) \geq 0, H_{k+i p+3}(X[k+3], X[k+4], \\
& \ldots, X[k+1], X[k+2]) \geq 0, \ldots, H_{k+i p+p}(X[k+p], \\
& X[k+1], \ldots, X[k+p-2], X[k+p-1]) \geq 0, \\
& \text { for all } i \geq 0\},
\end{aligned}
$$

and suppose that $\Omega_{k+1}^{\prime}$ is nonempty and can be defined by only a finite number of inequalities from (6). Then the following is a perfection of (4):

$\min J_{k+1}(z[k+1 \mid k+1])$

s.t. $z[k+1 \mid k+1] \in \Omega_{k+1}^{\prime}$.

In case $\Omega_{k+1}^{\prime}$ is empty for some $k$, then the corresponding optimization problem has no solution, and the ODRAP does not have a perfection. The reason for such an ill-conditioned ODRAP can be very complex. Wrong ODRAP formulations, or poor matching between the system dynamics and the constraints can lead to the nonexistence of PODRAP since the latter two are involved in the perfection process in Proposition 3. There do exist many energy problems which have a good matching between system dynamics and constraints and thus are PODRAP. The corresponding examples can be the dynamic economic dispatch problem (Xia et al., 2009), the water pumping system studied in Section 4, or any ODRAP satisfying Proposition 2 or Proposition 3.

\section{MPC approach to PODRAP}

Substituting the relations $X[k+1 \mid j]=X[k \mid j]+u_{k}^{j}$ into the $\operatorname{PODRAP}(k, k+p]$, one has:

$\min \widetilde{J_{k+1}}\left(X[k+1 \mid k+1], u_{k+1}^{k+1}, \ldots, u_{k+p-1}^{k+1}\right)$

s.t. $\quad \widehat{H_{k+1}}\left(X[k+1 \mid k+1], u_{k+1}^{k+1}, \ldots, u_{k+p-1}^{k+1}\right) \geq 0$.

If $X[k+1 \mid k+1]$ in (7) is substituted by a constant vector, then the optimization problem (7) has been reduced into a new optimization problem which has only $\left(u_{k+1}^{k+1}, \ldots, u_{k+p-1}^{k+1}\right)$ as its optimization variable. Denote this new optimization problem by $\operatorname{PODRAP}_{u}(k, k+p]$. Similarly, the substitution of $X[k+1 \mid k+1]$ by a constant vector in (1) leads to a new optimization problem with optimization variables $(X[k+2 \mid k+1], \ldots, X[k+p \mid k+1])$, denoted by $\operatorname{PODRAP}_{X}(k, k+p]$. Now the following MPC algorithm in control variable form is obtained.

Algorithm 1. Initialization Input $X[1 \mid 1]$, and let $k=0$.

(1) Measure $X[k+1 \mid k+1]$ and solve $\operatorname{PODRAP}_{u}(k, k+p]$ to find its optimal solution $u_{\mathrm{opt}}^{k}=\operatorname{col}\left(\left.u_{k+1}^{k+1}\right|_{\mathrm{opt}}, \ldots,\left.u_{k+p-1}^{k+1}\right|_{\text {opt }}\right)$;

(2) Implement $X[k+2 \mid k+2]=X[k+1 \mid k+1]+\left.u_{k+1}^{k+1}\right|_{\text {opt }}$ in the system, let $k=k+1$ and go to Step (1).

This algorithm is equivalent to the next algorithm in state variable form:

Algorithm 1'. Initialization Input $X[1 \mid 1]$, and let $k=0$.

(1) Measure $X[k+1 \mid k+1]$ and solve $\operatorname{PODRAP}_{X}(k, k+p]$ to find its optimal solution $z_{\mathrm{opt}}^{k}=\operatorname{col}\left(\left.X[k+2 \mid k+1]\right|_{\mathrm{opt}}, \ldots, X[k+\right.$ $\left.p \mid k+1]\left.\right|_{\text {opt }}\right)$

(2) Implement $X[k+2 \mid k+2]=\left.X[k+2 \mid k+1]\right|_{\text {opt }}$, let $k=k+1$ and go to Step (1).

The convergence of the MPC algorithm can be obtained by studying the following convex optimization problem:

$\min f(x)$

s.t. $x \in \Omega:=\{x: g(x) \geq 0, h(x)=0\}$, where $f$ is a smooth function from $\mathbb{R}^{n}$ to $\mathbb{R}^{1}, g$ and $h$ are vectorvalued smooth functions, $f$ is convex over the feasible domain $\Omega$ which is nonempty, bounded and convex.

The above convex optimization problem can be solved by the gradient method. The optimal solution $x_{\text {grad }}^{*}$ and the global minimum value $f\left(x_{\text {grad }}^{*}\right)$ are obtained. If the function is strictly convex, then this optimal solution $x_{\text {grad }}^{*}$ is also unique. In the following we will show that the same problem can also be solved by Algorithm 2.

Algorithm 2. Take a partition $\left(y^{1}, y^{2}, \ldots, y^{s}\right)$ for $x$ with $y^{j} \in$ $\mathbb{R}^{i_{j}-i_{j-1}}$ and $i_{0}=0$. Choose any initial value $x^{0}=\operatorname{col}\left(y_{0}^{1}, y_{0}^{2}, \ldots, y_{0}^{s}\right)$ $\in \Omega \subseteq \mathbb{R}^{n}$ and an error bound $\epsilon>0$, let Initial ${ }^{a}=y_{0}^{1}$, Initial $^{b}=$ $\operatorname{col}\left(y_{0}^{2}, \ldots, y_{0}^{s}\right), k=1, f_{\text {old }}^{*}=f\left(y_{0}^{1}, y_{0}^{2}, \ldots, y_{0}^{s}\right)$.

(i) Let $\tilde{y}=\operatorname{col}\left(y^{1}, \ldots, y^{k-1}, y^{k+1}, \ldots, y^{s}\right), F_{k}(\tilde{y}):=f\left(y^{1}, y^{2}, \ldots\right.$, $\left.y^{s}\right)\left.\right|_{y^{k}=\text { Initial }}{ }^{a}$, and solve the following problem

$$
\begin{array}{ll}
\min & F_{k}(\tilde{y}) \\
\text { s.t. } & \left.g\left(y^{1}, y^{2}, \ldots, y^{s}\right)\right|_{y^{k}=\text { Initial }^{a} \geq 0,} \geq 0, \\
& \left.h\left(y^{1}, y^{2}, \ldots, y^{s}\right)\right|_{y^{k}=\text { Initial }^{a}}=0,
\end{array}
$$

by the initial value $\tilde{y}=$ Initial $^{b}$. Denote the optimal solution by $\tilde{y}_{\text {new }}^{*}=\operatorname{col}\left(y_{*}^{1}, \ldots, y_{*}^{k-1}, y_{*}^{k+1}, \ldots, y_{*}^{s}\right)$.

(ii) If $F_{k}\left(\tilde{y}_{\text {new }}^{*}\right)<f_{\text {old }}^{*}-\epsilon$, then let $f_{\text {old }}^{*}=F_{k}\left(\tilde{y}_{\text {new }}^{*}\right), k=(k+$ 1) $\bmod s$, Initial ${ }^{a}=y_{*}^{k}$, Initial ${ }^{b}=\operatorname{col}\left(y_{*}^{1}, \ldots, y_{*}^{k-1}, y_{*}^{k+1}, \ldots\right.$, $\left.y_{*}^{s}\right)$ for the case $2 \leq k \leq s$ and Initial ${ }^{b}=\operatorname{col}\left(y_{*}^{2}, y_{*}^{3}, \ldots, y_{*}^{s}\right)$ for $k=1, x^{k}=\operatorname{col}\left(y_{*}^{1}, \ldots, y_{*}^{k-1}\right.$, Initial $\left.{ }^{a}, y_{*}^{k+1}, \ldots, y_{*}^{s}\right)$, and go to Step (i); otherwise stop the algorithm and return $k$, Initial ${ }^{a}$, Initial $^{b}, f_{\text {old }}^{*}$ and $x^{k}$.

Remark 1. Note that the above algorithm solves iteratively subproblems (9) to approach the solution of (8), each subproblem (9) has a lower dimension than the original problem (8). This algorithm does not specify any solution algorithm to solve the optimization problem in each iteration. Generally gradient based algorithms are good enough to compute these convex optimization problems. However, when uncertainties are considered, the optimization problems may not be convex and alternative algorithms such as genetic algorithms, particle swarm optimization, ant colony optimization, etc., from intelligent computing (Schrijver, 1998) can be very helpful.

Theorem 1. Fix the notations in problem (8) and a partition $x=$ $\operatorname{col}\left(y^{1}, y^{2}, \ldots, y^{s}\right)$, then Algorithm 2 solves problem (8) in the sense that its output $x^{k}$ satisfies $\lim _{k \rightarrow \infty} x^{k}=x_{0}^{*}$ for some $x_{0}^{*}$ and $f\left(x_{0}^{*}\right)=$ $f\left(x_{\text {grad }}^{*}\right)$. If $f$ is also strictly convex, then $x_{0}^{*}=x_{\text {grad }}^{*}$.

Proof. Note that $\Omega$ is bounded and the value of $f$ strictly decreases in Algorithm 2, this algorithm must converge to some point $x_{0}^{*}$, and it suffices to show that $f\left(x_{0}^{*}\right)$ equals $f\left(x_{\text {grad }}^{*}\right)$. Let $x_{0}^{*}=$ $\operatorname{col}\left(y_{*}^{1}, y_{*}^{2}, \ldots, y_{*}^{s}\right)$ and $x_{\text {grad }}^{*}=\operatorname{col}\left(y_{\text {grad }}^{1}, y_{\text {grad }}^{2}, \ldots, y_{\text {grad }}^{s}\right)$. Since Algorithm 2 stops at $x_{0}^{*}$, the point $\operatorname{col}\left(y_{*}^{1}, \ldots, y_{*}^{k-1}, y_{*}^{k+1}, \ldots, y_{*}^{s}\right)$ is a global optimal solution of the subproblem (9) for any $k=$ $1,2, \ldots, s$. By Theorem 3.4.3 of Bazaraa, Sherali, and Shetty (1993), we have $\left.\nabla F_{k}(\tilde{y})\right|_{x_{0}^{*}} \tilde{y} \geq 0$ for all $\tilde{y}$. In other words, we have $\left.\frac{\partial f}{\partial y^{1}}\right|_{x_{0}^{*}} y^{1}+\cdots+\left.\frac{\partial f}{\partial y^{k-1}}\right|_{x_{0}^{*}} y^{k-1}+\left.\frac{\partial f}{\partial y^{k+1}}\right|_{x_{0}^{*}} y^{k+1}+\cdots+\left.\frac{\partial f}{\partial y^{s}}\right|_{x_{0}^{*}} y^{s} \geq 0$. Note that the above inequality holds for all $k=1, \ldots, s$, and all $y^{1}, y^{2}, \ldots, y^{s}$, therefore, $\left.\nabla f\right|_{x_{0}^{*} X}=\left.\frac{\partial f}{\partial y^{1}}\right|_{x_{0}^{*}} y^{1}+\left.\frac{\partial f}{\partial y^{2}}\right|_{x_{0}^{*}} y^{2}+\cdots+$ $\left.\frac{\partial f}{\partial y^{s}}\right|_{x_{0}^{*}} y^{s} \geq 0$, where $x=\operatorname{col}\left(y^{1}, y^{2}, \ldots, y^{s}\right)$ is arbitrary. Again by Theorem 3.4.3 of Bazaraa et al. (1993), one has that $x_{0}^{*}$ is a global optimal solution of $(8)$, and $f\left(x_{0}^{*}\right)=f\left(x_{\text {grad }}^{*}\right)$. When $f$ is strictly convex, the solution is unique and thus $x_{0}^{*}=x_{\mathrm{grad}}^{*}$. 
The proof of the convergence of Algorithm $1^{\prime}$ follows from the observation that there is a one-to-one correspondence between the loops in Algorithm $1^{\prime}$ and the loops in Algorithm 2. For instance, consider the first loop of Algorithm $1^{\prime}$ for $k=0$. Given $X[1 \mid 1]=X_{0}$, the algorithm aims to find the minimum value of $J_{1}\left(X_{0}, X[2 \mid 1], X[3 \mid 1], \ldots, X[p+1 \mid 1]\right)$ under the constraints $H_{1}\left(X_{0}, X[2 \mid 1], X[3 \mid 1], \ldots, X[p+1 \mid 1]\right) \geq 0$. Now consider the first loop of applying Algorithm 2 to the PODRAP. Fix the partition $z[1 \mid 1]=\operatorname{col}\left(y^{1}, y^{2}, \ldots, y^{p}\right)$ with $y^{i}:=X[i \mid 1], i=1, \ldots, p$. Take Initial $^{a}=X_{0}$, the optimization problem in Step (i) of Algorithm 2 is the same as that in Algorithm $1^{\prime}$, which establishes the one-to-one correspondence.

Theorem 2. Let $z^{*}$ be the globally optimal solution of the PODRAP $(0, p], z_{\mathrm{opt}}^{k}=\operatorname{col}\left(\left.X[k+2 \mid k+1]\right|_{\mathrm{opt}}, \ldots,\left.X[k+p \mid k+1]\right|_{\mathrm{opt}}\right)$, the optimal solution of the $k$-th loop in Algorithm $1^{\prime}$ which is obtained under the initial value $X[k+1 \mid k+1]=X_{\text {initial }}^{k}$, and $X_{\text {initial }}^{k}=$ $\left.X[k+1 \mid k]\right|_{\text {opt }}$ for $k \geq 1$. Suppose $k=p k_{1}+r_{1}$ with $0 \leq r_{1}<p$. Denote $\bar{z}^{k}=\operatorname{col}\left(\left.X\left[p k_{1}+p+1 \mid k+1\right]\right|_{\mathrm{opt}}, \ldots, X\left[p k_{1}+p+r_{1} \mid k+\right.\right.$ $\left.1]\left.\right|_{\mathrm{opt}}, X_{\text {initial }}^{k},\left.X\left[p k_{1}+r_{1}+2 \mid k+1\right]\right|_{\mathrm{opt}}, \ldots,\left.X\left[p k_{1}+p \mid k+1\right]\right|_{\mathrm{opt}}\right)$ for the case $r_{1} \geq 1$, and $\bar{z}^{k}=\operatorname{col}\left(X_{\text {initial }}^{k},\left.X[k+2 \mid k+1]\right|_{\text {opt }}, \ldots, X[k+\right.$ $\left.p \mid k+1]\left.\right|_{\text {opt }}\right)$ for $r_{1}=0$; then $\lim _{k \rightarrow \infty} J_{k}\left(\bar{z}^{k}\right)=J_{1}\left(z^{*}\right)$. If, furthermore, the functions $J_{1}$ and $H_{1}$ are strictly convex, then the optimal solution of each loop in Algorithm 1' is unique, and therefore $\lim _{k \rightarrow \infty} \bar{z}^{k}=z^{*}$.

Now consider the robustness of Algorithm 1'. For convenience, we consider only the case that uncertainty happens in the execution of the controller or the measurement of states, that is, consider $X[k+2 \mid k+2]=X[k+1 \mid k+1]+\left.u_{k+1}^{k+1}\right|_{\text {opt }}+w_{k+1}$ or equivalently $X[k+2 \mid k+2]=\left.X[k+2 \mid k+1]\right|_{\text {opt }}+w_{k+1}$, where $w_{k+1}$ is a disturbance vector satisfying $\left\|w_{k+1}\right\|<e$ and $e$ is a constant. By a similar reason as the proof of convergence of the MPC algorithm, the robustness can be shown by proving the robustness of Algorithm 2. To this end, let Initial ${ }^{a}=y_{*}^{k}+w_{*}^{k}$ in Step (ii) of Algorithm 2, where $w_{*}^{k}$ is a disturbance vector satisfying $\left\|w_{*}^{k}\right\|<e$

Theorem 3. Fix the notations in problem (8) and a partition $x=$ $\operatorname{col}\left(y^{1}, y^{2}, \ldots, y^{s}\right)$, and assume that $\|\nabla f(x)\| \leq L$ for a constant $L$ for all $x \in \Omega$. Let $x_{\text {grad }}^{*}$ be the optimal solution of problem (8) obtained by gradient method; $\epsilon$ the error bound defined in Algorithm 2; $c$ a positive constant which is less than $\epsilon$, the constant disturbance $w_{*}^{k}$ satisfying $\left\|w_{*}^{k}\right\|<e$; Initial ${ }^{a}=y_{*}^{k}+w_{*}^{k}$ executed in Step(ii) of Algorithm 2; and e small enough so that $e<\min \{c / L,(\epsilon-c) / L\}$; then there exists an integer $N_{0}$ such that for any $k>N_{0}$, the output $x^{k}$ of the $k$-th loop in Algorithm 2 belongs to the domain $\Omega^{\prime}:=\left\{x:\left\|x-x_{\text {grad }}^{*}\right\|<c\right\}$.

Proof. Consider the $k$-th loop in Algorithm 2. Step (i) gives an optimal solution $\tilde{y}_{\text {new }}^{*}=\operatorname{col}\left(y_{*}^{1}, \ldots, y_{*}^{k-1}, y_{*}^{k+1}, \ldots, y_{*}^{s}\right)$ for Initial ${ }^{a}$ equals some vector $\xi^{k}$. Denote $z_{k}=\operatorname{col}\left(y_{*}^{1}, \ldots, y_{*}^{k-1}, \xi^{k}, y_{*}^{k+1}, \ldots\right.$, $\left.y_{*}^{s}\right)$, and suppose $F_{k}\left(\tilde{y}_{\text {new }}^{*}\right)<f_{\text {old }}^{*}-\epsilon$. Define $\bar{z}_{k}=\operatorname{col}\left(y_{*}^{1}, \ldots\right.$, $\left.y_{*}^{k-1}, \xi^{k}, y_{*}^{k+1}+w_{*}^{k}, y_{*}^{k+2}, \ldots, y_{*}^{s}\right)$. In the $(k+1)$-th loop, denote the optimal solution of step (i) by $\operatorname{col}\left(\tilde{y}_{*}^{1}, \ldots, \tilde{y}_{*}^{k}, \tilde{y}_{*}^{k+2}, \ldots, \tilde{y}_{*}^{s}\right)$, and define similarly $z_{k+1}=\operatorname{col}\left(\tilde{y}_{*}^{1}, \ldots, \tilde{y}_{*}^{k}, y_{*}^{k+1}+w_{*}^{k}, \tilde{y}_{*}^{k+2}, \ldots, \tilde{y}_{*}^{s}\right)$ and the corresponding $\bar{z}_{k+1}$ under the disturbance $w_{*}^{k+1}$.

Without loss of generality suppose the three points $\bar{z}_{k}, z_{k}$, and $z_{k+1}$ are different from each other. Obviously $f\left(\bar{z}_{k}\right)<f\left(z_{k+1}\right)$, and $\delta_{k}:=f\left(\bar{z}_{k}\right)-f\left(z_{k+1}\right)>0,\left|f\left(z_{k}\right)-f\left(\bar{z}_{k}\right)\right|=|\nabla f|_{\xi}\left(z_{k}-\bar{z}_{k}\right) \mid \leq$ $\left\|w_{*}^{k}\right\|\left\|\left.\nabla f\right|_{\xi}\right\| \leq e L$. Note that if $\delta_{k}<c$, then $\left|f\left(z_{k}\right)-f\left(z_{k+1}\right)\right| \leq$ $\left|f\left(z_{k}\right)-f\left(\bar{z}_{k}\right)\right|+\left|f\left(\bar{z}_{k}\right)-f\left(z_{k+1}\right)\right|<c+e L<\epsilon$, and Algorithm 2 stops. This implies that $z_{k}$ is treated as $x_{\text {grad }}^{*}$, thus $\left|f\left(\bar{z}_{k}\right)-f\left(x_{\text {grad }}^{*}\right)\right|=$ $\left|f\left(\bar{z}_{k}\right)-f\left(z_{k}\right)\right| \leq e L<c$, and $\bar{z}_{k}$ enters the domain $\Omega^{\prime}$. If $\delta_{k} \geq c$, then $f\left(z_{k}\right)-f\left(z_{k+1}\right)=f\left(z_{k}\right)-f\left(\bar{z}_{k}\right)+f\left(\bar{z}_{k}\right)-f\left(z_{k}\right)>\delta_{k}-e L \geq c-e L>0$.

Repeat the above steps for all the $k$, then either $f\left(\bar{z}_{k}\right)$ enters the domain $\Omega^{\prime}$, or $f\left(z_{k}\right)$ continues decreasing. Note that the problem is over a bounded convex domain, therefore in the latter case $f\left(z_{k}\right)$ converges to $f\left(x_{\text {grad }}^{*}\right)$. Then by a similar analysis as done for the case $\delta_{k}<c, f\left(\bar{z}_{k}\right)$ will eventually enter the domain $\Omega^{\prime}$.

A similar procedure as the proof of the convergence of the MPC algorithm shows the following robustness result.

Corollary 1. Suppose $\Omega_{\text {PODRAP }}:=\left\{\operatorname{col}(X[1], \ldots, X[p+1]): H_{1}\right.$ $(X[1], \ldots, X[p+1]) \geq 0\}$ is the feasible domain of the problem $\operatorname{PODRAP}(0, p], z^{*}$ is the globally optimal solution of $\operatorname{PODRAP}(0, p]$, the norm of the gradient of the cost function $J_{1}$ of $\operatorname{PODRAP}(0, p]$ has the upper bound $L$ on $\Omega_{\mathrm{PODRAP}}, z_{\mathrm{opt}}^{k}=\operatorname{col}(X[k+2 \mid k+$ $\left.1]\left.\right|_{\text {opt }}, \ldots,\left.X[k+p \mid k+1]\right|_{\text {opt }}\right)$ is the optimal solution of the $k$-th loop in Algorithm $1^{\prime}$ which is obtained under the initial value $X[k+$ $1 \mid k+1]=X_{\text {initial }}^{k}$, and $X_{\text {initial }}^{k}=\left.X[k+1 \mid k]\right|_{\text {opt }}+w_{k}$ for $k \geq 1$. Assume also that $\epsilon$ is a small enough positive constant, $c$ is a positive constant which is less than $\epsilon$, the disturbance $w_{k}$ satisfies $\left\|w_{k}\right\|<e, e$ is small enough so that $e<\min \{c / L,(\epsilon-c) / L\}, k=p k_{1}+r_{1}$ with $0 \leq r_{1}<p$, and define $\bar{z}^{k}$ as Theorem 2 , then $\left\|\bar{z}^{k}-z^{*}\right\|<c$.

Remark 2. In a practical energy system, the end-user demand will often be approximately periodic which corresponds to the case that disturbances happen in some coefficients in the function $H_{1}$. Since the optimal solution of the convex optimization problem depends smoothly on the corresponding coefficients in $H_{1}$, the MPC algorithm is also robust against the disturbances in the demand.

\section{A case study on a water pumping system}

Voluntary load shedding (VLS), or the so-called strategic offer, is a scheme proposed by a South African electricity supplier to solve the serious electricity shortage problem (Zhang, Xia, \& Alexander, 2008). In Zhang et al. (2008), the optimal scheduling of a pumping system with 21 pumps in terms of VLS is solved with an open loop control approach. In this case study, this pumping system is reconsidered to illustrate the MPC approach. In order to simplify the model, it is assumed that all the water from the 21 pumps flows to a big reservoir instead of the 4 reservoirs in Zhang et al. (2008). The VLS model over a $24 \mathrm{~h}$ period with $24 \times 21=504$ number of variables and 101 constraints can be summarized as follows.

$$
\begin{array}{ll}
\min & \sum_{i=1}^{21}\left[\sum_{j=1}^{24} c_{j} P_{i} u_{i j}-q \sum_{\text {peak }} P_{i}\left(1-u_{i \ell}\right)\right] \\
\text { s.t. } & \sum_{i=1}^{21} V_{i} u_{i j} \geq D_{j}, \quad R^{\min } \leq R_{k} \leq R^{\max }, \\
& 0 \leq u_{i j} \leq 1, \quad \sum_{i=1}^{21} P_{i} u_{i j} \leq P_{\max }, \\
& \sum_{i=1}^{21} V_{i} u_{i j} \leq V_{\text {supply }}, \\
& \sum_{\ell \in \text { peak }} \sum_{i=1}^{21} P_{i} u_{i \ell} \leq r P_{\max }, \\
1 \leq j \leq 24, \quad 2 \leq k \leq 24,
\end{array}
$$

where $u_{i j} \in[0,1]$ is the switching status of the $i$-th pump at the $j$-th time interval. Peak means the index $\ell$ in the summation varies in the peak tariff time intervals. The electricity price at time $j$ is $c_{j}, q$ is the incentive constant and $V_{i}$ denotes the volume of water that the $i$-th pump can pump within one hour when the pump is working at its maximum power $P_{i} . R^{\mathrm{min}}$ and $R^{\mathrm{max}}$ are constants representing the capacity of the reservoir, $R_{k}$ is the water level of the reservoir at time $k, R_{1}=R^{\min }, P_{\max }$ is the specified maximum electricity demand. $V_{\text {supply }}$ is a constant denoting the incoming water supply rate (Mega-liter per hour, or $\mathrm{Ml} / \mathrm{h}$ ), and $r$ is the percentage so that a percentage $(1-r)$ of the maximal possible total load during peak hours is shed. 


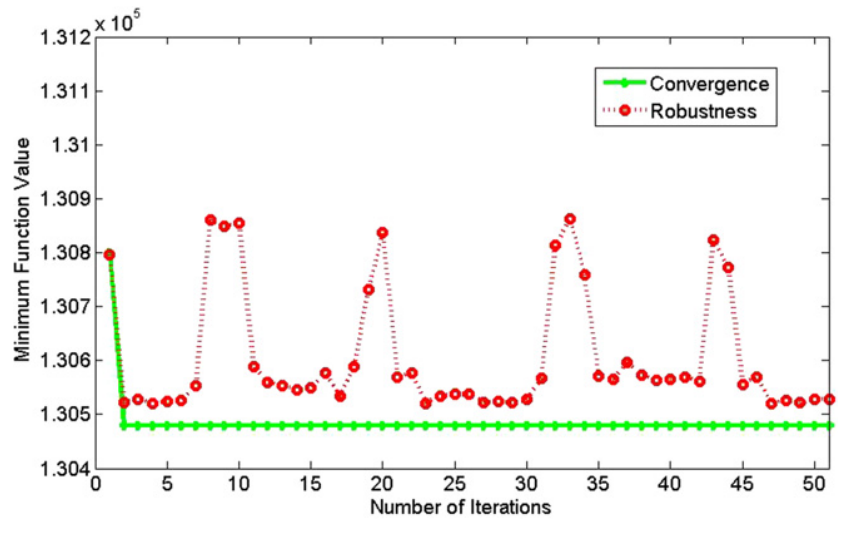

Fig. 1. Convergence and robustness.

This is an ODRAP. By Proposition 3, a PODRAP is obtained by adding the missing constraints $\sum_{i=1}^{21} V_{i}\left(u_{i 24}+\cdots+u_{i 2}+u_{i 1}\right) \leq$ $R^{\max }-R^{1}+\left(D_{1}+D_{2}+\cdots+D_{24}\right)$. For this PODRAP, MPC Algorithm 1 converges to the global minimum $1.3048 \times 10^{5}$ of $\operatorname{VLS}(0,24]$ at the second loop (see the solid line in Fig. 1). As for the robustness of the MPC algorithm, assume that $u_{\text {opt }}[k \mid k]$ in Step (2) of Algorithm 1 is replaced by $u_{\text {opt }}[k \mid k]+w[k]$, where $w[k]$ is a noise vector and each of its components is generated randomly by the Matlab uniform distribution function rand(1)/50. The minimum objective function value varies within a small neighborhood of the global minimum value $1.3048 \times 10^{5}$ as is clear from the dotted line in Fig. 1. If the first iteration step is excluded, then Fig. 1 shows that the maximum error happens at the 8 -th step. The relative error of $0.29 \%$ is quite satisfactory.

\section{Conclusions}

This paper introduces the perfect optimal dynamic resource allocation problem and provides a corresponding MPC algorithm to solve the periodic implementation problem for the optimal solution. The convergence and robustness of the MPC algorithm are proved. This establishes a close connection of an MPC solution and a solution of a global optimization problem. An application of the MPC approach to the voluntary load shedding problem for a water pumping system illustrates the convergence and robustness of this MPC algorithm.

\section{Acknowledgements}

We would like to thank the anonymous reviewers for their valuable comments, and Mrs. Mathilda du Preez for text editing this paper.

\section{References}

Aissi, H., Bazgan, C., \& Vanderpooten, D. (2009). Min-max and min-max regret versions of combinatorial optimization problems: a survey. European Journal of Operational Research, 197(2), 427-438.

Allgöwer, F., \& Zheng, A. (2000). Nonlinear model predictive control. Berlin: Birkhauser Verlag.

Bazaraa, M. S., Sherali, H. D., \& Shetty, C. M. (1993). Nonlinear programming theory and algorithms. New York: John Wiley \& Sons.

Belfares, L., Klibi, W., Lo, N., \& Guitouni, A. (2007). Multi-objectives Tabu search based algorithm for progressive resource allocation. European Journal of Operational Research, 177, 1779-1799.

Biegler, L. T., \& Zavala, V. M. (2009). Large-scale nonlinear programming using IPOPT: an integrating framework for enterprise-wide dynamic optimization. Computers and Chemical Engineering, 33, 575-582.
Chang, T. S., \& Seborg, D. E. (1983). A linear programming approach to multivariable feedback control with inequality constraints. International Journal of Control, 37, 583-597.

Ferrari-Trecate, G., Gallestey, E., Letizia, P., Spedicato, M., Morari, M., \& Antoine, M (2004). Modeling and control of co-generation power plants: a hybrid system approach. IEEE Transactions on Control Systems Technology, 12, 694-705.

Garcia, C. E., Prett, D. M., \& Morari, M. (1989). Model predictive control: theory and practice-a survey. Automatica, 25(3), 335-348.

Ibaraki, T., \& Katoh, N. (1988). Resource allocation problems: algorithmic approaches. Cambridge: The MIT Press.

Lee, S., Kumara, S., \& Gautam, N. (2007). Efficient scheduling algorithm for component-based network. Future Generation Computer Systems, 23 558-568.

Lee, J. H., \& Lee, J. M. (2006). Approximate dynamic programming based approach to process control and scheduling. Computers and Chemical Engineering, 30, $1603-1618$.

Middelberg, A., Zhang, J., \& Xia, X. (2009). An optimal control model for load shiftingwith application in the energy management of a colliery. Applied Energy, 86, $1266-1273$.

Munawar, S. A., \& Gudi, R. D. (2005). A multilevel, control-theoretic framework for integration of planning, scheduling, and rescheduling. Industrial \& Engineering Chemistry Research, 44, 4001-4021.

Pasadyn, A. J., Lee, H., \& Edgar, T. F. (2008). Scheduling semiconductor manufacturing processes to enhance system identification. Journal of Process Control, 18, 946-953.

Patriksson, M. (2008). A survey on the continuous nonlinear resource allocation problem. European Journal of Operational Research, 185, 1-46.

Qin, S. J., \& Badgwell, T. A. (2003). A survey of industrial model predictive control technology. Control Engineering Practice, 11, 733-764.

Schrijver, A. (1998). Theory of linear and integer programming. New York: John Wiley \& Sons.

van Staden, A. J., Zhang, J., \& Xia, X. (2009). A model predictive control strategy for load shifting in a water pumping scheme with maximum demand charges. In IEEE Bucharest PowerTech.

Xia, X., Zhang, J., \& Elaiw, A. (2009). A model predictive control approach to dynamic economic dispatch problem. In IEEE Bucharest PowerTech.

Zadeh, L. A., \& Whalen, B. H. (1962). On optimal control and linear programming. IRE Transactions on Automatic Control, 7(4), 45-46.

Zafra-Cabeza, A., Ridao, M. A., \& Camacho, E. F. (2008). Using a risk-based approach to project scheduling: a case illustration from semiconductor manufacturing. European Journal of Operational Research, 190, 708-723.

Zhang, J., Xia, X., \& Alexander, D. (2008). Demand side optimal strategy for voluntary load shedding. In The second IASTED Africa conference on power and energy systems.

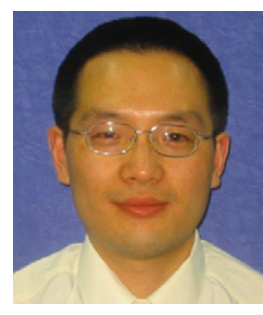

Jiangfeng Zhang obtained his B.Sc. and Ph.D. in computing mathematics from Xi'an Jiaotong University, China, in July 1995 and December 1999 respectively. From February 2000 to August 2002 he was a lecturer at the Shanghai Jiaotong University, China. Then he was a postdoctoral researcher in the Chinese University of Hong Kong, Ecole Centrale de Nantes (France), Nanyang Technological University (Singapore), University of Liverpool (UK), and University of Pretoria (RSA). He has been a senior lecturer and then an associate professor in the University of Pretoria from November 2008. He is a certified energy manager and a certified measurement and verification professional. His current research interests are energy management and control theory.

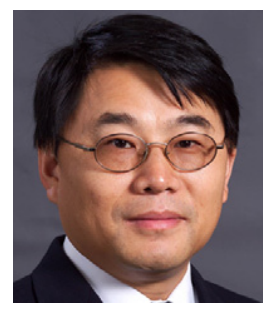

Xiaohua Xia is a professor in the Electrical, Electronic and Computer Engineering at the University of Pretoria, South Africa, director of the Centre of New Energy Systems, and the director of the National Hub for the Postgraduate Programme in Energy Efficiency and Demand-side Management. He was academically affiliated with the University of Stuttgart, Germany, the Ecole Centrale de Nantes, France, and the National University of Singapore before joining the University of Pretoria in 1998. Prof. Xia is a fellow of the Institute for Electronic and Electrical Engineers (IEEE), a fellow of the South African Academy of Engineering (SAAE), and he has an A rating from the South African National Research Foundation (NRF). He also serves as the chair of the Technical Committee of Non-linear Systems of the International Federation of Automatic Control (IFAC). He has been an associate editor of Automatica, IEEE Transactions on Circuits and Systems II, IEEE Transactions on Automatic Control, and specialist editor (control) of the SAIEE Africa Research Journal. 